\title{
Discussion on rapid evaluation method of large- scale land degradation in China
}

\author{
Yu Xing ${ }^{*}$, Yingjie Zhou, Weiling Yao, Hao Wang, Li Li, and Jie Wang \\ China Aero Geophysical Survey and Remote Sensing Center for Natural Resources, 100083 Beijing, \\ China
}

\begin{abstract}
This paper discusses the effects of land degradation assessment based on the index of net primary productivity (NPP), rain use efficiency of vegetation (RUE) and relative rain use efficiency of vegetation(rRUE). The results show that the land degradation evaluation method based on the correlation coefficient of NPP with time and the annual average and annual maximum of rRUE can comprehensively reflect the status of land productivity. It plays a good role in indicating the effect of land degradation, and is more conducive to remote sensing quantitative evaluation and rapid monitoring of large-scale land degradation.
\end{abstract}

\section{Forward}

The traditional land degradation assessment method is to carry out field investigation or laboratory records of soil at a specific point in a specific area. This method is suitable for monitoring and evaluation of point level or small regional scale and less field investigation, but not suitable for large-scale remote sensing monitoring of land degradation. With the development of remote sensing and GIS technology, it is possible to realize mesoscale or large-scale remote sensing evaluation and monitoring of land degradation in time and space, and the idea of land degradation assessment and monitoring technology based on "3s" is becoming more and more clear. However, most of them obtain land type information through visual interpretation of remote sensing images, and then combine with ground survey to semi-quantitatively evaluate land degradation and its impact. Due to regional differences and multi-source data, there are a variety of land degradation indicators; classification standards are not consistent; standardized quantitative indicators are lacking; and most of them are indirect indicators, which are difficult to obtain through remote sensing technology. There are few studies on large-scale rapid quantitative assessment of land degradation. Accurately obtaining soil information and vegetation information by remote sensing, and monitoring the changes of various soil and vegetation indicators by remote sensing in the process of land degradation is the basis of remote sensing quantitative evaluation and monitoring. Using NPP and RUE indicators, scholars have achieved good results in monitoring the scope and degree of land degradation. For example: Yin He et al.[1] selected the RUE evaluation index of high-temporal resolution of remote sensing inversion and used the time series analysis method to evaluate the development trend of

*Corresponding author:63216429@qq.com 
desertification in Inner Mongolia Autonomous Region. Wang Hongyan[2] carried out remote sensing monitoring and evaluation of land degradation in the wind and sand source areas of Beijing and Tianjin based on NPP and RUE. Gao Zhihai et al.[3] discussed the remote sensing evaluation method for desertification based on RUE. In this paper, three indexes suitable for large-scale net primary productivity (NPP), rain use efficiency of vegetation(RUE), relative rain use efficiency of vegetation (rRUE) are compared and analyzed, and the optimal method for evaluating land degradation is obtained.

\section{Comparison of evaluation methods}

- Calculate the $R U E$, that is, the ratio of $N P P$ to precipitation $(P)$ :

$$
R U E=N P P / P
$$

- The annual moisture index (MI) from 2000 to 2010 is calculated, and the climatic regions are divided at fixed intervals $(0.15$ in this paper) to form a climatic gradient zone (18 climatic zones are divided in this paper).

- According to the probability of the RUE value in each type of climatic zone divided by the MI, the RUE values corresponding to the $95 \%$ and $5 \%$ lower quantile of the RUE probability distribution are selected as the upper limit value RUE0.95 and the lower limit value RUE0.05, which can better express the maximum and minimum values of relative land production capacity in each climatic zone.

- According to the maximum value of RUE, the minimum value of RUE in each type of climate gradient zone, and the median value of MI interval in each type of climate gradient zone, the fitting function equation, namely the edge function model, is established.

- Taking the spatial raster data of MI as independent variables, the spatial raster data of the maximum and minimum values of RUE in China are obtained by spatial simulation according to the edge function model.

- The rRUE is calculated, and the threshold analysis is used to grade the land production capacity of rRUE. The $r R U E$ calculation formula is

$$
r R U E=\left(R U E-R U E_{0.05}\right) /\left(R U E_{0.95}-R U E_{0.05}\right) .
$$

In the formula, $r R U E$ represents the position of each pixel RUE within the range of the maximum and minimum potential RUE in the climate gradient, and between $[0,1] ; R U E_{0.05}$ and $R U E_{0.95}$ represent the minimum and maximum values of potential rain use efficiency, respectively.

- The spatial distribution maps of rRUE in different periods are generated. The rRUE maps eliminates the influence of large-scale climate gradient. The RUE edge function is the natural "benchmark" of vegetation potential productivity in the climate region. Therefore, any land cover type in the region can be directly compared.

- Annual MI refers to the ratio of annual precipitation $(P)$ to potential evapotranspiration $(P E)$ in the same period [4].

\section{Results and analysis}

The spatial distribution maps of annual average NPP and annual RUEmean in China are calculated, as shown in Fig.1. The spatial distribution maps of rRUEmean and rRUEmax in China are calculated, as shown in Fig.2. 

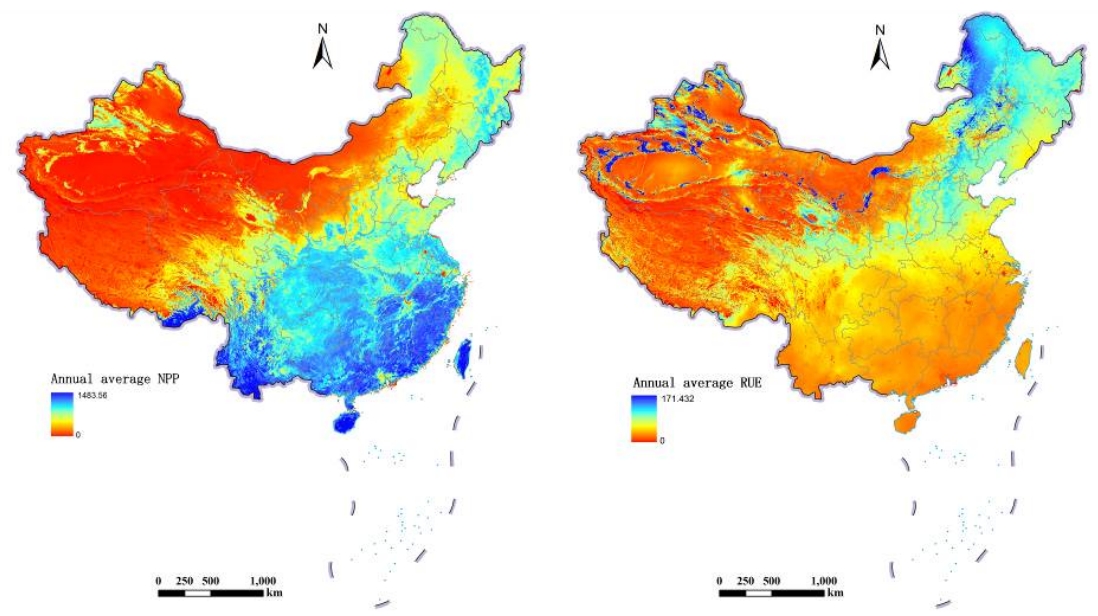

Fig. 1. Spatial distribution of annual average NPP (left) and annual RUE (right) in recent 10 years.
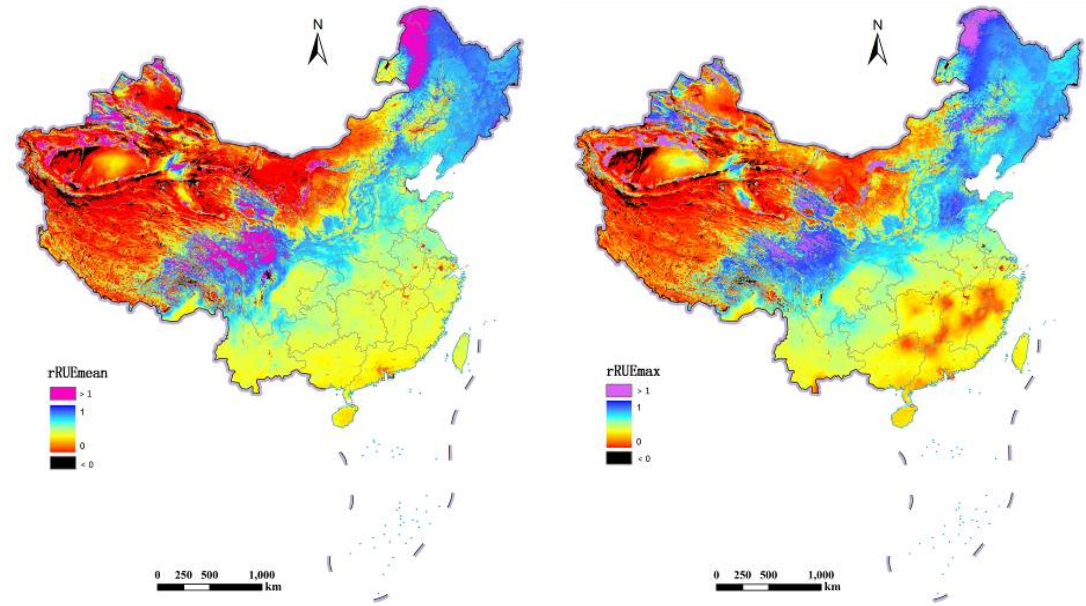

Fig. 2. Spatial distribution of rRUEmean (left) and rRUEmax (right) in recent 10 years.

From the analysis of the calculation results of NPP and RUE, the spatial distribution of RUE in China is very different from that of NPP. In southeast China, the annual NPP value is high, but because the precipitation in this region is also abundant, the RUE value is low. In northeast China, because the vegetation NPP is significantly smaller than that in the southeast, and because the difference in precipitation is smaller than that in NPP, the RUE value in the northeast is higher. In northwest China, there are some areas where the NPP value is low, but the RUE value is high, which is due to the small precipitation. Although the vegetation productivity shows an upward trend and the productivity has increased, it does not mean that the land condition in this area has improved, nor does it mean that the land has not been degraded. When the vegetation productivity shows a downward trend, it can not fully show that the land is degraded, perhaps due to the reduction of precipitation. The actual situation of the land has not become worse.

Although both NPP and RUE indicators play an indicative role in the evaluation of land degradation, through the test and analysis of the change trend correlation analysis methods of NPP and RUE, it can be found that the statistical data of the two indicators reflect different land degradation trends. According to the analysis of spatial distribution, the change trends of NPP and RUE have no obvious law, some changes are opposite, or some 
changes are consistent. Therefore, the two indicators of NPP and RUE in the evaluation of land degradation need to be improved and need to be used comprehensively.

Land degradation is relative to the initial or previous state, which involves the benchmarking of land degradation. The "benchmark" of land degradation assessment refers to the maximum potential state that the ecosystem can achieve under certain climatic conditions [5], that is the final stable state that can be achieved by local natural vegetation without human disturbance [6]. RUE is a comprehensive indicator of ecosystem vegetation productivity and health. NPP and RUE represent the status of vegetation productivity. They are two important indicators to measure land productivity and effectively represent the development trend of land degradation. However, RUE is essentially a ratio. Obviously, if the vegetation productivity of the 2 areas is the same, but their different climatic conditions, the RUE value in arid areas with less annual precipitation is higher. Therefore, for such a large range of China, there is an obvious climatic gradient, which cannot be directly compared with RUE. Instead, the climate impact needs to be removed and the index values of different climate gradient zones can be compared on the basis of "relative benchmark".

On the basis of considering the climate "relative benchmark", the significance test of the correlation coefficient of NPP with time and the annual average and annual maximum of rRUE are used to comprehensively reflect the status of land productivity, which has a good indicative effect on land degradation.

\section{Conclusion}

The land production capacity is evaluated by multi-year average rRUE and annual maximum rRUE, and the time series NPP change is used to monitor vegetation degradation. Finally, considering the results of land production capacity evaluation and vegetation degradation monitoring, the average status of land degradation in China is evaluated. The rRUE of this method can comprehensively reflect the status of land productivity, has a good indication of land degradation, can reflect the objective situation of land degradation, and is more conducive to remote sensing quantitative evaluation and rapid monitoring of largescale land degradation.

\section{Acknowledgements}

This paper is supported by Programs of China Geological Survey (Remote sensing monitoring of national mining exploration and ecological spatial in key areas).

\section{References}

1. H. Yin, Z.G. Li, Y.L. Wang, F. Cai, Acta Geographica Sinica,66,653 (2011)

2. H.Y. Wang. Assessment and monitoring of land degradation using NPP and vegetation rain use efficiency,Chinese Academy of Forestry (2013)

3. Z.H. Gao, Z.Y. Li, G.D. Ding, L. Y. Li, Science of Soil and Water Conservation, 3, 37 (2005)

4. S.W. Zhuang, H.C. Zuo, P.C. Ren, G. J. Xiong, B. D. Li, W. C. Dong, L. Y. Wang, Climatic and Environmental Research, 18,617(2013)

5. P.P. Mi. The designing research on land degradation monitoring system, Northeast Agricultural University(2008)

6. Y.P. Liu, Journal of Arid Land Resources and Environment, 12,74(1998) 